\title{
O USO POTENCIAL DAS TECNOLOGIAS DA INFORMAÇÃO E COMUNICAÇÃO NOS PROCESSOS COMUNICACIONAIS DA FORMAÇÃO EM SEGURANÇA PÚBLICA: PERSPECTIVAS E DESAFIOS PARA UMA ATUAÇÃO EM REDE
}

\author{
LAURO DE FREITAS/BA MAIO/2018 \\ Emanuel do Rosário Santos Nonato - UNEB - enonato@uneb.br \\ Maria da Soledade Cardoso Landim Batista - UNEB - maria.landim79@gmail.com \\ Mary Valda Souza Sales - UNEB - marysales@uneb.br
}

Tipo: Investigação Científica (IC)

Natureza: Relatório Final de Pesquisa

Categoria: Métodos e Tecnologias

Setor Educacional: EDUCAÇÃO CORPORATIVA

\begin{abstract}
RESUMO
O Estudo trata da reflexão sobre as Tecnologias da Informação e Comunicação na formação em Segurança Pública, com o objetivo de compreender que uso das TIC favorece a conformação de uma rede cooperativa no processo formativo dos Soldados da Polícia Militar da Bahia. Para tanto, a análise estabelece o Estudo de Caso como método para o alcance dos objetivos propostos. Os resultados obtidos indicam o uso instrumental das TIC para efeito de controle, por meio de comunicação unidirecional, entre os órgãos envolvidos na formação dos Soldados PM. Em contrapartida, foi observada a viabilidade de utilização do potencial comunicativo das TIC, para favorecer a integração, diálogo e cooperação desse sistema. A pesquisa apontou a possibilidade de constituição de rede cooperativa, com a inserção do uso potencial das TIC nos processos comunicativos no CFSd PM, resguardando funções e responsabilidades, bem como a hierarquia e disciplina, princípios da PMBA.
\end{abstract}

Palavras-chave: TIC. Rede Cooperativa. Formação - Segurança Pública 


\section{INTRODUÇÃO}

O debate sobre as Tecnologias da Informação e Comunicação (TIC) vem se tornando cada vez mais frequente em diversas áreas do conhecimento, tendo em vista as múltiplas possibilidades de uso e aplicação de seus potenciais; mas, estudos têm avançado, especialmente, em razão dos impactos que têm promovido na forma de comunicação e de aproximação entre as pessoas. Assim, um dos desafios da atualidade é justamente pensar em novas formas de interligar e compartilhar informações e experiências, a partir das potencialidades que as TIC oferecem, para transformar 0 modo como as pessoas interagem e aprendem.

Por isso, é fundamental pensar em novas estratégias formativas, apoiadas nos potenciais das TIC, que cumpram suas finalidades de planejar, acompanhar e avaliar as ações educacionais, de forma dinâmica, horizontalizada, participativa e integrada, para mobilizar os esforços de maneira efetiva, com vista ao alcance dos objetivos educacionais, inclusive em estruturas organizacionais como a da Polícia Militar da Bahia (PMBA), alicerçadas nos pilares da hierarquia e da disciplina

Neste caso, o estudo buscou analisar o uso das TIC na formação em segurança pública, observando seus efeitos e possibilidades nos processos comunicativos ocorridos durante a formação dos Soldados PM (Sd PM), curso realizado pela Polícia Militar (PM) para preparar os que ingressam nas fileiras da corporação.

O Curso de Formação de Soldados PM (CFSd PM) é considerado um curso técnico, com duração, em média, de nove meses, realizado por uma estrutura composta por órgãos consultivos, diretivos e operacionais da PMBA, cuja finalidade vai desde 0 estabelecimento de diretrizes à execução do curso propriamente dito, que ocorre nos chamados Núcleos de Ensino (NE), distribuídos na capital e interior do estado, cuja escolha varia a cada edição do curso, de acordo com a quantidade de alunos.

Essa estrutura flutuante produz efeitos no desenvolvimento do processo formativos dos Soldados PM e demanda, em certa medida, que o sistema de ensino da PMBA, formalmente estruturado, atue em rede. Considerando tais implicações, esta investigação ocorre no sentido de responder à questão: de que forma o uso das TIC nos processos comunicativos potencializa o desenvolvimento de uma rede cooperativa no Curso de Formação de Soldados da PMBA?

$\mathrm{Na}$ busca da compreensão e construção de um posicionamento ante essa indagação, toma-se como principal objetivo desse estudo investigar e compreender como a 
utilização das TIC pode contribuir para o desenvolvimento de uma rede cooperativa no Curso de Formação de Soldados da PMBA, explorando os potenciais comunicacionais disponíveis à interação dos responsáveis pelo acompanhamento e desenvolvimento do curso.

\section{REFERENCIAL TEÓRICO}

O principal fundamento deste estudo se baseia na ampliação do conceito de TIC, de forma a não reduzir seu uso à eficácia e alcance de seus aparatos tecnológicos ou à qualidade das informações veiculadas através dos canais comunicativos, ainda que reconheça a importância desses aspectos subsidiariamente.

Nesse sentido, Hetkowski sugere que:

As TIC são compreendidas como surgimento de uma outra articulação de linguagens, encarnada em novos suportes que são as máquinas com as quais os homens se comunicam, dotando-as da capacidade de processarem e intercambiarem informações (HETKOWSKI, 2004, p. 17)

Dessa forma, ainda que recorra também às máquinas como forma de executar sua capacidade comunicacional, as TIC oferecem uma possibilidade de articular novas estratégias e linguagens, que ampliem o processo de troca de informações. Pois, a articulação favorece a inserção de novas conexões, de forma a intensificar o fluxo de informações, potencializando o processo de construção e difusão de saberes e experiências.

Por isso, é preciso destacar que o desafio da ação comunicativa não reside nos meios disponíveis ou na aquisição de recursos comunicativos sofisticados; mas está na possibilidade de interligação, conexão e diálogo, apoiada pelo potencial comunicativo das TIC, pois:

(...) com o advento das Tecnologias da Informação e Comunicação (TIC), as quais, entre outras coisas, imprimem um caráter comunicativo e informacional à coisa tecnológica, de modo que trazem em si, simultaneamente, um elemento comum e basilar de qualquer processo tecnológico, e se particularizam e diferenciam por sua qualidade comunicativa e informacional. (LIMA JÚNIOR, 2012, p. 44)

O caráter comunicativo e informacional dos aparatos tecnológicos, potencializado pelas TIC, eleva o processo dialógico e interativo para outro patamar. E essa condição não 
está relacionada diretamente a uma conexão on-line, mas vincula-se à natureza social do processo comunicacional que, por si só é dinâmico (LIMA JÚNIOR, 2012, p. 44), buscando estabelecer laços, relações.

Apesar de apontar, especificamente, para os movimentos sociais, é interessante considerar a análise de Castells (2013), sobre a possibilidade de um padrão emergente dos movimentos sociais em rede, observando que:

O uso das redes de comunicação da internet e dos telefones celulares é essencial, mas a forma de conectar-se em rede é multimodal. Inclui redes sociais on-line e off-line, assim como redes preexistentes e outras formadas durante as ações do movimento. Formam-se redes dentro do movimento (...). As tecnologias que possibilitam a constituição de redes são significativas por fornecer a plataforma para essa prática continuada e que tende a se expandir, evoluindo com a mudança do formato do movimento. (CASTELLS, 2013, p. 163-164)

A conexão apresentada transcende um modelo unilateral, considerando seu movimento, suas dinâmicas. Ela é rica pela diversidade de (inter)relação que apresenta e não segue apenas em uma única direção ou modo, limitando suas perspectivas.

Assim, a comunicação deve ocorrer no sentido de mediar, interagir, e não apenas com a intenção de informar, direcionar, ordenar, coordenar; pois, agindo dessa maneira o diálogo entre as partes não é fluído, partindo de um polo gerador para as pontas do sistema, num único movimento de ida, deixando de explorar o potencial de uma atuação articulada em rede.

Nesse sentido, o diálogo se destaca como estratégia fundante e fundamental nessa dinâmica comunicativa, de forma a construir uma "via de mão-dupla", na circulação dos saberes em rede, que não apenas atinge os seus diversos pontos, mas integra-os, articula-os.

Nesse entendimento, a pedagogia das redes, sugere a comunicação e o diálogo como seus elementos essenciais, indicando também a interligação como principal desafio. Pois, se a conexão é peculiar à rede, sua efetivação está na cooperação entre seus agentes, de forma a operar junto.

Dessa forma, “(...) a colaboração pode ser entendida como uma filosofia de vida e a cooperação como uma interação projetada para facilitar a realização do trabalho, da atividade e da tarefa" (SALES. 2013). Assim, é possível pensar em trabalho cooperativo 
em rede, mesmo em estruturas organizacionais verticalizadas, marcadas essencialmente pelos princípios da hierarquia e disciplina, como a PMBA.

A intenção de refletir sobre a possibilidade de realização de trabalho em rede na corporação policial militar baiana surge em decorrência do atual modelo formativo adotado pela PMBA, configurado por um sistema de ensino próprio responsável pela preparação de seus integrantes, através da realização de cursos de formação, estágios, treinamentos, aperfeiçoamento, habilitação, adaptação, enfim diversos processos e atividades educativas para os profissionais de segurança pública, conforme os objetivos, estrutura e carga horária disponível para cada ação.

Uma estrutura de ensino atualmente composta pelos seguintes órgãos da PMBA: Instituto de Ensino e Pesquisa, Academia de Polícia Militar, Centro de Formação e Aperfeiçoamento de Praças, Batalhões e Núcleos de Ensino, além dos Colégios da Polícia Militar, situados na capital e interior do estado, conforme legislação específica.

Por isso, um dos principais desafios na realização do Curso de Formação de Soldados $\mathrm{PM}$, nos moldes atuais, é o aprimoramento de seu acompanhamento e desenvolvimento, buscando uma atuação mais articulada e integrada, especialmente no apoio ao Núcleos de Formação, sediados em unidades operacionais, pois não são fixos, variando a cada edição do CFSd PM, de acordo com a quantidade de profissionais aprovados nos concursos públicos.

\section{PROCEDIMENTOS METODOLÓGICOS}

A pesquisa se concentrou nas ações de acompanhamento e desenvolvimento do processo formativo em Segurança Pública dos candidatos à função de Soldados PM, promovido pelos Núcleos de Ensino, responsáveis pela realização do Curso de Formação de Soldados PM, ocorrido no ano de 2014 (CFSd PM/ 2014).

Foi escolhida a primeira edição do CFSd PM no ano de 2014, considerando a dimensão desse processo formativo, ocorrido em 23 Núcleos de Ensino, situados na capital e interior do estado baiano. Assim, para acesso aos dados referentes ao objeto deste estudo, questionários foram enviados nos meses de outubro e novembro do ano de 2015 aos policiais militares que atuaram na função de Instrutor-chefe dos Batalhões de Ensino, Instrução e Capacitação (BEIC) e dos Núcleos de Ensino (NE), que sediaram o Curso de Formação de Soldados PM, ocorrido entre os dias 06 de janeiro de 2014 e 19 de setembro de 2014 , contando com 1300 policiais militares e 95 bombeiros militares aprovados. 
A finalidade do estudo mostra a extensão do exercício de análise e compreensão a serem desenvolvidas, de modo que os esforços precisam ser organizados para o alcance dos resultados desejados. Assim, a escolha pelo Estudo de Caso obedece aos parâmetros que o objeto oferece, dialogando com as suas especificidades, pois, "como método de pesquisa, o estudo de caso é usado em muitas situações, para contribuir ao nosso conhecimento dos fenômenos individuais, grupais, organizacionais, sociais, políticos e relacionados" (YIN, 2015, p. 4).

\section{APRESENTAÇÃO E DISCUSSÃO DOS RESULTADOS}

A proposta de incentivo ao trabalho em equipe nas ações de acompanhamento e desenvolvimento do CFSd PM, apoiada nos preceitos da cooperação e potencializada pelo uso das TIC, surge como alternativa à atuação isolada e fragmentada do sistema de ensino PM, nos moldes atuais.

A comunicação que é estabelecida no desenvolvimento do CFSd PM dá-se de maneira diretiva e polarizada, ou seja, não funciona de forma dialógica; ou seja, as ações comunicativas fazem apenas o movimento de ida, de um ponto a outro desse sistema e há indícios de sobreposições de pontos na origem do envio dessas informações.

Isso porque o diagnóstico colhido a partir do trabalho de pesquisa apresenta o uso da TIC na formação profissional dos Soldados PM direcionado ao controle do curso. Com isso, o esforço é destinado à aquisição de equipamentos tecnológicos e direcionamento unilateral da comunicação no CFSd PM.

Assim, como superação da atual dinâmica comunicativa, o desenho da proposta do processo comunicativo na formação PM, a partir da possibilidade de auxílio mútuo, apoiada na ideia de uma rede cooperativa se apresenta da seguinte forma:

Figura - Rede bidirecional na Formação dos Soldados PM 


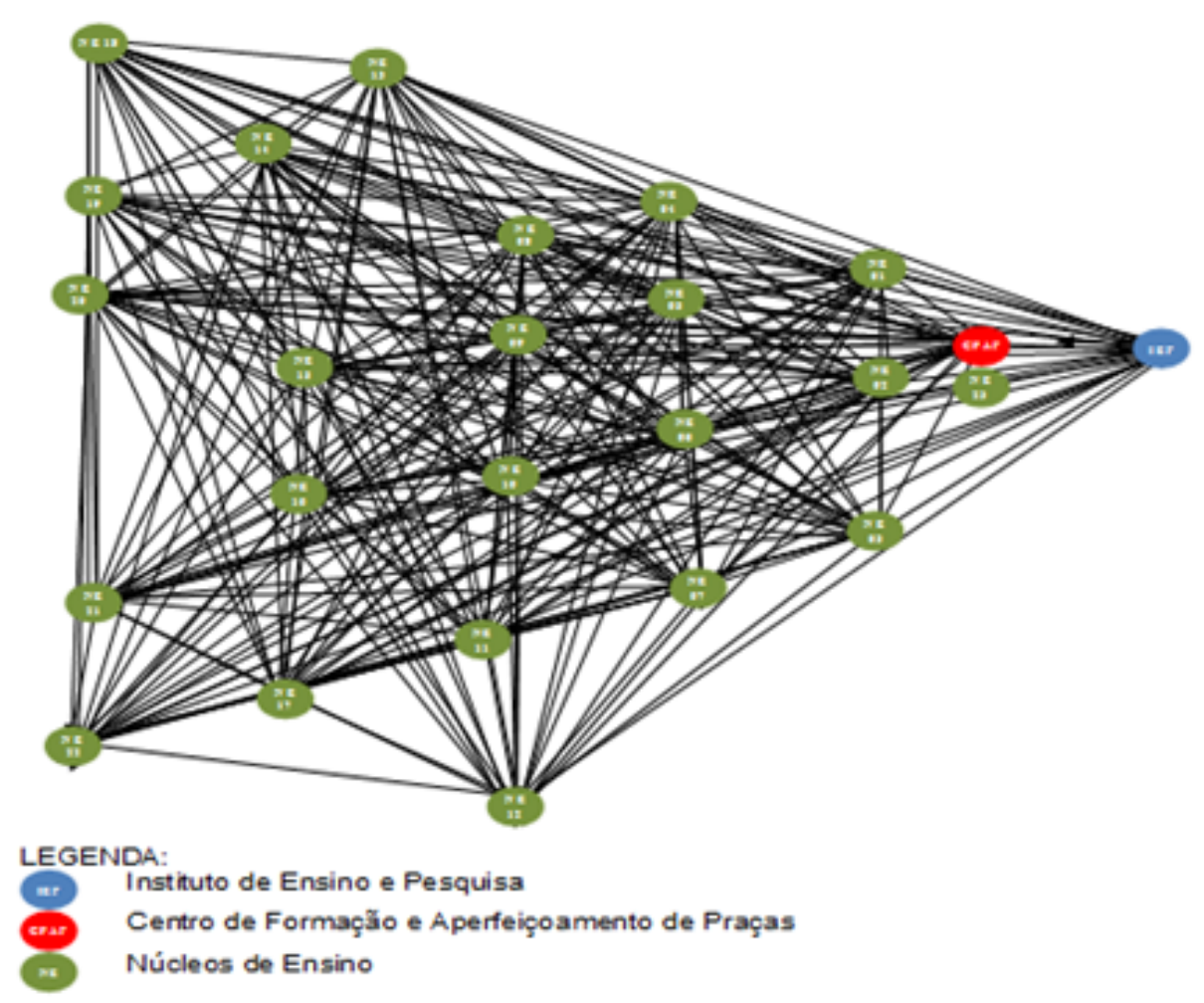

Fonte: Autores.

A principal mudança nesse desenho é na apresentação de um esquema de comunicação bidirecional. O centro não perde seu protagonismo; mas, em razão de não dispor de tempo e alcance necessários para se retroalimentar e movimentar a rede, cede espaço de atuação, em regime cooperativo, aos Núcleos de Ensino, nos quais, efetivamente, os problemas ocorrem e requerem uma intervenção imediata.

Pois, o principal entendimento é da necessidade de atuação conjunta dos integrantes da rede cooperativa, no limite de suas funções e responsabilidades, mas que permita a todos acompanhar, efetivamente, o que ocorre no processo formativo e intervir para superar os desafios que se apresentam ao longo das etapas de planejamento, desenvolvimento e avaliação do curso e alcançar os objetivos do processo formativo.

Nesse sentido, o potencial comunicativo das TIC se apoia, principalmente, nas relações de troca e compartilhamento de informações e experiências, de maneira cooperativa, tendo em vista o fortalecimento do coletivo por um lado, mas, por outra via, buscando reconhecer e considerar o indivíduo e respeitar sua autonomia e diversidade. 
comunicativo da formação dos Soldados PM, uma forma alternativa de comunicação, apoiada no uso das TIC, que atue em uma perspectiva de rede e supere o atual esquema unidirecional do CFSd PM, de modo a promover processos dialógicos e cooperativos, cujas ocorrências foram identificadas no campo de pesquisa.

Nesse ponto, as TIC se apresentam como estratégia fundamental, pois favorece 0 compartilhamento de experiências e informações entre os órgãos responsáveis pelo desenvolvimento do CFSd PM. Em decorrência, espera-se impactar o processo educativo através de possibilidades geradas pela exploração do potencial comunicativo das TIC.

A principal consideração nesse caso é a preservação dos papéis e responsabilidades, fundamentados na hierarquia e disciplina PM. Afinal, a ideia de trabalho em rede no CFSd PM é amparada por outro princípio estatutário, a cooperação, que orienta a articulação do sistema, de modo a permitir a aproximação entre todos envolvidos na realização do curso, incentivando a solidariedade entre seus integrantes.

Por isso, a cooperação no âmbito educacional, como construção coletiva do conhecimento (SALES, 2013), favorece o trabalho em comum, pois possibilita uma atuação integrada e em rede, sem necessariamente ocorrer a ruptura de estruturas hierarquizadas, como acontece em regimes colaborativos. Ou que admita apenas uma participação isolada dos seus integrantes, deixando de explorar o potencial de articulação desse sistema.

\section{CONSIDERAÇÕES FINAIS}

A discussão das TIC e do seu potencial comunicativo no contexto da formação de Soldados da PMBA apresentou-se, desde o começo do percurso desta pesquisa, como um desafio, pois sinalizava para a necessidade de debater o processo comunicativo na formação PM, apoiado pelo uso das TIC. Mas, era também uma possibilidade de promover a análise de uma temática pouco explorada no cenário acadêmico, pois abordava de questões relacionadas à formação daqueles que estão ingressando na Corporação PM, para assumir as funções de Soldado.

O cenário apresentado indica que, apesar de estar formalmente estruturado, com definição específica de papéis e funções, o sistema de ensino PM não atua em rede. Esse aspecto não compromete, essencialmente, o seu funcionamento, mas pode dificultar a troca de informações e experiências entre os diversos pontos da estrutura educacional responsável pelo funcionamento dos cursos de formação. 
Assim, foi possível pensar em novas estratégias de atuação nos processos formativos profissionais em Segurança Pública, de forma dinâmica e articulada, sem ir de encontro aos pilares institucionais da hierarquia e disciplina. Isso porque, no caso específico da PMBA, existe a previsão legal de desenvolvimento permanente do espírito de cooperação, isto é, "operar junto", resultando na possibilidade de uma ação conjunta e, de fato, integrada desse sistema educacional.

O esforço, contudo, não é apenas de obtenção de instrumentos tecnológicos para construir canais de comunicação, a fim de "facilitar" o acompanhamento e desenvolvimento do CFSd PM. A ampliação do entendimento das TIC mostra que apenas a aquisição desses aparatos não resulta em ações interativas; é preciso uma mudança de postura e perspectiva, que possibilite a conexão e participação de todos envolvidos no acompanhamento da formação de Soldados PM, na medida de seus papéis e responsabilidades.

Dessa forma, esse estudo cumpre sua finalidade de investigar e compreender como a utilização das TIC pode contribuir para o desenvolvimento de uma rede cooperativa no Curso de Formação de Soldados da PMBA, proposta em seu objetivo geral. Pois, o potencial comunicativo das TIC se apresenta como uma alternativa para a conformação de uma rede cooperativa no CFSd PM, de forma a unir e integrar os pontos do sistema de ensino da PMBA, promovendo um ambiente de cooperação mútua, de troca de informações e experiências, especialmente entre os responsáveis pelas ações de acompanhamento e desenvolvimento do Curso de Formação de Soldados, realizado pelo Centro de Formação e Aperfeiçoamento de Praças, situado na capital, bem como nos Batalhões de Ensino, Instrução e Capacitação e Núcleos de Ensino, localizados no interior baiano.

\section{REFERÊNCIAS}

CASTELLS, M. Redes de indignação e esperança: movimentos sociais na era da internet. Trad. Carlos Alberto Medeiros. 1 ed. Rio de Janeiro: Zahar, 2013.

HETKOWSKI, T. M. Políticas Públicas: Tecnologias da Informação e Comunicação e Novas Práticas Pedagógicas. Tese (Doutorado) - Doutorado em Educação (FACED/UFBA), Faculdade de Educação, Universidade Federal da Bahia, Salvador, 2004.

LIMA JR, A. S. de. O dinamismo do sujeito na ciência. In: LIMA JR, A. S. de (Org.). Educação e Contemporaneidade: contextos e singularidades. Salvador, EDUFBA: 
EDUNEB, 2012.

SALES, M. V. S. Tessituras entre mediação e autoria nas práticas de currículo na formação a distância: a construção do conhecimento no contexto universitário $281 f$. 2013. Tese (Doutorado) - Doutorado em Educação (FACED/UFBA), Faculdade de Educação, Universidade Federal da Bahia, Salvador, 2013.

YIN, R. K. Estudos de caso: planejamento e métodos. Porto Alegre: Bookman, 2005. 